\title{
Pattern formation in reaction-diffusion models with spatially inhomogeneous diffusion coefficients
}

\author{
Philip K. Maini, Debbie L. Benson, and Jonathan A. Sherratt \\ Centre for Mathematical Biology, Mathematical Institute, 24-29 St. Giles', \\ Oxford $O X 13 L B, U K$
}

[Received 20 May 1992 and in revised form 10 September 1992]

\begin{abstract}
Reaction-diffusion models for biological pattern formation have been studied extensively in a variety of embryonic and ecological contexts. However, despite experimental evidence pointing to the existence of spatial inhomogeneities in various biological systems, most models have only been considered in a spatially homogeneous environment. The authors consider a two-chemical reaction-diffusion mechanism in one space dimension in which one of the diffusion coefficients depends explicitly on the spatial variable. The model is analysed in the case of a step function diffusion coefficient and the insight gained for this special case is used to discuss pattern generation for smoothly varying diffusion coefficients. The results show that spatial inhomogeneity may be an important biological pattern regulator, and possible applications of the model to chondrogenesis in the vertebrate limb are suggested.
\end{abstract}

Keywords: diffusion-driven instability; pattern isolation; inhomogeneous environment; developmental hierarchy.

\section{Introduction}

Reaction-diffusion theory for pattern formation was first proposed by Turing (1952) in his seminal work on the chemical basis for morphogenesis. Turing demonstrated that, in certain systems, diffusion could give rise to spatial inhomogeneities (Turing structures) in the concentration of reacting chemicals (termed morphogens). With the hypothesis that cells differentiate according to local morphogen concentration, this prepattern in morphogen concentration would then be translated into a spatial pattern of cell differentiation. This is the fundamental concept underlying the application of reaction-diffusion models to morphogenesis: for a review see, for example, the books by Meinhardt (1982) and Murray (1989). Although experimental evidence for the existence of morphogens in biological systems is, at present, tenuous, Turing structures have recently been found in chemical systems (Castets et al., 1990; Ouyang \& Swinney, 1991).

An alternative model for pattern formation is the gradient model proposed by Wolpert $(1969,1981)$ and applied to skeletal patterning in the vertebrate limb. The basic hypothesis of this model is that a gradient in morphogen concentration is set up along the anterior-posterior (AP) axis of the developing limb bud. With the further hypothesis that there are a series of threshold levels for differentiation, this model exhibits patterns which are consistent with those observed in chondrogenesis in the limb. However, although gradients in the concentration of certain retinoids 
have been shown to exist in the developing chick limb (Thaller \& Eichele, 1987), their role in pattern formation remains controversial (Wanek et al., 1991; Noji et al., 1991). Moreover, although positional information in the gradient model is set up by a simple mechanism, its interpretation is via a complex series of arbitrary thresholds for which detailed mechanisms have not been proposed. In contrast, the mechanism of spatial patterning in Turing systems, although much more complex, is well understood, and its interpretation is via a simple single threshold level.

Turing models have been proposed as possible pattern generators in a variety of biological situations. However, in the particular application to the developing limb bud there are a number of drawbacks to this model (Maini \& Solursh, 1991). Firstly, it has been shown that solutions of Turing models are sensitive to initial conditions (Bard \& Lauder, 1974) and that to generate the sequence of bifurcations observed in skeletal patterning in the limb requires the parameters of the system to evolve in a very precise and complex manner (Dillon et al., 1992). These properties are obvious drawbacks of a model for a robust patterning mechanism such as that in the limb. Recently, however, it has been shown that the choice of mixed boundary conditions can greatly influence the patterning properties of the Turing model and one can, in fact, generate the sequence of bifurcations observed in the limb bud in a robust and simple manner (Dillon et al., 1992).

Another criticism of Turing systems is that the patterns produced are scale dependent. In the developing chick limb, recent experiments (Wolpert \& Hornbruch, 1990) show that a recombinant limb bud consisting of the anterior halves of two different limb buds develops two humeri, yet is the same size as a normal limb bud, which only produces one humerus. This clearly contradicts the standard Turing model which predicts an identical number of elements in limbs of the same size.

A further criticism of Turing models for limb development is that they produce spatial patterns which are symmetric along the AP axis of the limb bud. The skeletal elements in the limb, however, are not symmetrically situated along this axis (see e.g. Walbot \& Holder, 1987: Chap. 18). Moreover, the patterns generated by the standard Turing model cannot, in general, distinguish between distinct skeletal rudiments such as the different types of digit, because the amplitude of oscillations is constant throughout the solution profile.

The reason for these inconsistencies with experimental observation may be that most applications of Turing systems assume that the background environment is spatially homogeneous, that is, all model parameters are assumed constant across the domain. However, there is experimental evidence to suggest that in some biological systems environmental inhomogeneity may be an important regulator of pattern. This was recognized by Turing himself, who, in his 1952 paper, wrote that 'most of an organism, most of the time, is developing from one pattern into another, rather than from homogeneity into a pattern'. In this paper we consider how an underlying spatial prepattern in diffusion coefficients modifies the pattern forming properties of a reaction-diffusion model. In developmental biology, spatial variation in morphogen diffusivity may be controlled by the concentration of a regulatory chemical which, for example, affects morphogen transport by binding to the morphogen or by modulating gap junction permeability (Othmer \& Pate, 1980; Hunding \& Sørenson, 1988; Brümmer et al., 1991). Several authors have investigated 
numerically the effects of spatially varying reaction terms in reaction-diffusion systems (Gierer \& Meinhardt, 1972; Hunding et al., 1990; Lacalli, 1990) and the effects of spatially varying diffusion (Hunding, 1987, 1989). Auchmuty \& Nicolis (1974) and Herschkowitz-Kaufman (1975) analyse a system in which one of the reaction terms depends explicitly on space. However, we are not aware of any analytical studies of the effects of spatially inhomogeneous diffusion coefficients.

We present a two-step reaction-diffusion model for pattern formation. The first step establishes a prepattern in diffusion coefficients which then modifies the pattern-forming properties of the overlying reaction-diffusion mechanism. Such a system is not mathematically tractable in general, but can be analysed when the spatial variation in diffusion coefficients is piecewise constant. In Section 2 we present a linear analysis for a system in which one of the diffusion coefficients is a step function in space with only one point of discontinuity, while the other is constant across the domain. In Section 3 we present numerical solutions of the full nonlinear model and show that the predictions from linear analysis compare well with the solutions of the nonlinear problem. We also show that the model can exhibit highly asymmetric and isolated patterns. Although a discontinuity in diffusion coefficient is biologically implausible, this simple case provides an understanding of how spatial inhomogeneities can modulate pattern in more realistic systems. In Section 4 we consider a more biologically realistic two-step model in which the spatially heterogeneous distribution of a regulatory chemical sets up a smooth gradient in one of the diffusion coefficients of the reaction-diffusion model. This composite model essentially incorporates the gradient model with the classical Turing model. We discuss the possible application of our composite model to chondrogenesis in Section 5.

\section{Linear analysis}

A reaction-diffusion system is said to exhibit diffusion-driven instability (Turing, 1952) if a uniform steady state is stable to spatially homogeneous perturbations, but unstable to spatially inhomogeneous perturbations. When diffusive instability is possible, stable spatially heterogeneous solutions of the model equations may exist. Therefore, our primary interest is to determine the parameter domain in which diffusion-driven instability may occur, and we begin by summarizing necessary and sufficient conditions for the generation of pattern in a homogeneous domain.

We consider the standard dimensionless two-species Turing model in one space dimension:

$$
\begin{aligned}
& \frac{\partial u}{\partial t}=\gamma f(u, v)+\frac{\partial^{2} u}{\partial x^{2}} \\
& \frac{\partial v}{\partial t}=\gamma g(u, v)+D \frac{\partial^{2} v}{\partial x^{2}}
\end{aligned}
$$

Here $\gamma$ is a scale factor proportional to the length of the domain, and $f$ and $g$ are the reaction kinetics for which there exists a positive homogeneous steady state $\left(u_{0}, v_{0}\right)$. In this model it is assumed that morphogen diffusivity is constant throughout 
the domain, so that the ratio $D$ of diffusion coefficients is a positive constant. We further assume that the spatial domain has been nondimensionalized to $[0,1]$, and we impose zero-flux boundary conditions. Standard linear analysis shows that diffusion-driven instability may occur in this system for parameters lying in the Turing space defined by

$$
\begin{aligned}
a+d & <0, \\
a d & >b c, \\
a+D d & >0 \\
(a+D d)^{2} & >4 D(a d-b c),
\end{aligned}
$$

where

$$
a=\left.\frac{\partial f}{\partial u}\right|_{\left(\nu_{0}, \nu_{0}\right)}, \quad b=\left.\frac{\partial f}{\partial v}\right|_{\left(\nu_{0}, v_{0}\right)}, \quad c=\left.\frac{\partial g}{\partial u}\right|_{\left(\mu_{0}, v_{0}\right)}, \quad d=\left.\frac{\partial g}{\partial v}\right|_{\left(\mu_{0}, \nu_{0}\right)} .
$$

For a detailed derivation of these results, see, for example, the book by Murray (1989). The above results hold in higher dimensions but in the rest of this paper we restrict our attention to one dimension. The one-dimensional geometry facilitates analytical investigation and, moreover, for the particular application to the limb bud discussed below, this is a relevant approximation and is widely used (see, for a review, Maini \& Solursh, 1991). It follows from $(2.2 \mathrm{a}-\mathrm{c})$ that both $a d$ and $b c$ must be negative, so that, to within an arbitrary relabelling of species, there are essentially two kinds of reaction-diffusion models capable of exhibiting diffusion-driven instability, having kinetic matrices taking one of the following forms:

$$
\left[\begin{array}{ll}
+ & - \\
+ & -
\end{array}\right]
$$$$
\left[\begin{array}{ll}
+ & + \\
- & -
\end{array}\right]
$$

pure activator-inhibitor model

cross activator-inhibitor model

In the former case, the steady-state solutions are always in phase, whereas those in the latter are in antiphase, at least in the vicinity of primary bifurcation points (Dillon et al., 1992). In both cases, along primary bifurcation branches, the model solutions are characterized by an approximately equal spaced succession of peaks and troughs of constant amplitude spread across the domain (Fig. 1), such that increases in the length of the domain lead, in general, to more complicated patterns with a greater number of peaks and troughs. An important property of these solutions is the sensitivity of pattern polarity to initial conditions. This may be seen analytically for the linearized system, for whenever $(\hat{u}, \hat{v})=\left(u-u_{0}, v-v_{0}\right)$ is a solution of

$$
\hat{u}_{t}=f_{u} \hat{u}+f_{v} \hat{v}+\hat{u}_{x x}, \quad \hat{v}_{t}=g_{u} \hat{u}+g_{v} \hat{v}+D \hat{v}_{x x}
$$

then $(-\hat{a},-\hat{v})$ is also a solution. The solution of the linearized model is therefore given by either $u=u_{0}+\hat{u}, v=v_{0}+\hat{v}$ or $u=u_{0}-\hat{u}, v=v_{0}-\hat{v}$. The actual solution is determined by the initial conditions, so that different sets of initial conditions may determine solutions with opposite polarity. This multiplicity of solutions is also exhibited by the full nonlinear system.

We now outline the derivation of the conditions for diffusion-driven instability in an inhomogeneous environment in which one of the diffusion coefficients is a step 


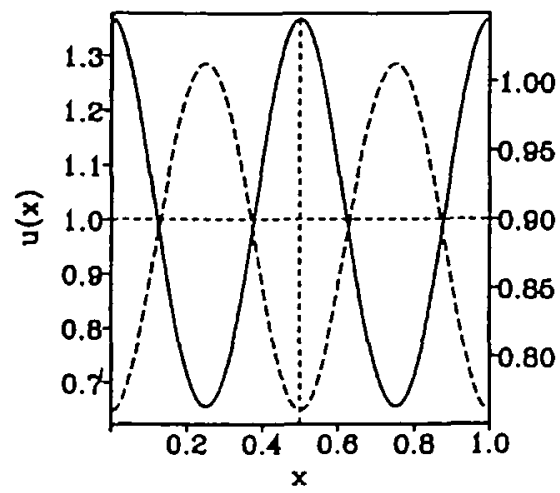

(a)

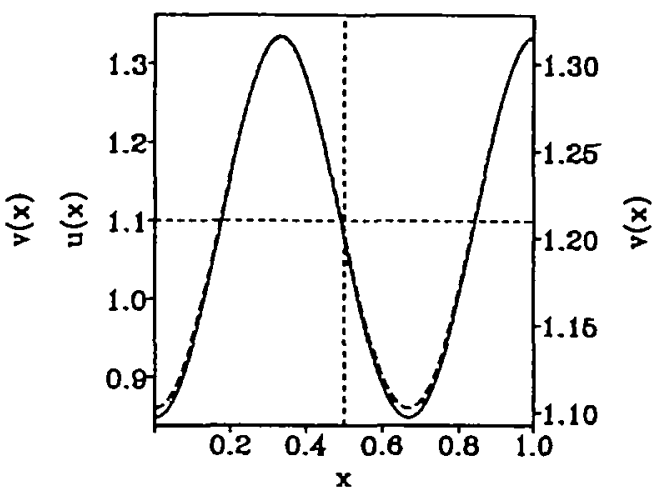

(b)

FIG. 1. (a) and (b) show, respectively, typical steady-state solution profiles of a cross activator-inhibitor model (Gierer \& Meinhardt, 1972) and a pure activator-inhibitor model (Schnackenberg, 1979). In (a) the morphogen concentration profiles $u$ and $v$ are approximately in antiphase, in (b) they are in phase. In both cases the horizontal line (---) shows the position of the homogeneous steady state ( $u$ : $-; 0:---)$.

function in space with a single point of discontinuity (see Benson et al., 1992, for full details). In particular, we consider the model equations (2.1) with the diffusion term in (2.1b) replaced by $(\partial / \partial x)[D(x) \partial v / \partial x]$, where

$$
D(x)= \begin{cases}D^{-} & (0 \leqslant x<\xi), \\ D^{+} & (\xi<x \leqslant 1)\end{cases}
$$

$D^{-} \neq D^{+}$, and $\xi \in(0,1)$. Clearly, the conditions for the steady state to be stable to spatially homogeneous perturbations are the same as in the case of homogeneous environment $\left(D^{-}=D^{+}\right)$, that is $(2.2 \mathrm{a})$ and $(2.2 \mathrm{~b})$. To derive the analogues of $(2.2 \mathrm{c})$ and $(2.2 \mathrm{~d})$ for the inhomogeneous case, we linearize the model about the steady state and look for solutions to the linear system of the form $u-u_{0}=(\exp \lambda t) X_{u}(x)$, $v-v_{0}=(\exp \lambda t) X_{v}(x)$. Substituting these expressions into the linearized model gives rise to a pair of coupled ordinary differential equations in $X_{w}$ and $X_{v}$ :

$$
\begin{aligned}
X_{u}^{\prime \prime}+(a-\lambda) X_{u}+b X_{v} & =0, \\
\left(D(x) X_{u}^{\prime}\right)^{\prime}+c X_{u}+(d-\lambda) X_{v} & =0,
\end{aligned}
$$

where a prime denotes $\partial / \partial x$.

Since our model divides the domain into two parts on which the diffusivity of $u$ is the same but that of $v$ is different, we initially consider equations (2.6) on the interval $[0, \xi)$. On this interval, adding (2.6a) to $s_{i}^{-} / D^{-}$times (2.6b) for $i=1,2$, leads to the pair of equations

$$
\left(X_{u}+s_{i}^{-} X_{v}\right)^{n}+\left(a-\lambda+\frac{c s_{i}^{-}}{D^{-}}\right)\left(X_{u}+\frac{b+(d-\lambda) s_{i}^{-} / D^{-}}{a-\lambda+c s_{i}^{-} / D^{-}} X_{v}\right)=0,
$$

where $s_{1}$ and $s_{2}$ are chosen to be the roots of the quadratic

$$
c s^{2}+\left[D^{-}(a-\lambda)-(d-\lambda)\right] s-b D^{-}=0 .
$$


This reduces (2.7) to two equations in $X_{u}+s_{l}^{-} X_{v}$ which may be solved subject to the zero-flux boundary conditions at $x=0$ to give

$$
\begin{aligned}
& X_{u}(x)=\frac{1}{s_{2}^{-}-s_{1}^{-}}\left(\frac{\left(\Gamma_{u}+s_{1}^{-} \Gamma_{v}\right) s_{2}^{-}}{\cos \xi \alpha_{1}^{-}} \cos \alpha_{1}^{-} x-\frac{\left(\Gamma_{u}+s_{2}^{-} \Gamma_{v}\right) s_{1}^{-}}{\cos \xi \alpha_{2}^{-}} \cos \alpha_{2}^{-} x\right), \\
& X_{v}(x)=\frac{1}{s_{2}^{-}-s_{1}^{-}}\left(\frac{\Gamma_{u}+s_{2}^{-} \Gamma_{v}}{\cos \xi \alpha_{2}^{-}} \cos \alpha_{2}^{-} x-\frac{\Gamma_{u}+s_{1}^{-} \Gamma_{v}}{\cos \xi \alpha_{1}^{-}} \cos \alpha_{1}^{-} x\right) .
\end{aligned}
$$

Here $x \in[0, \xi), \alpha_{i}^{-}=a-\lambda+c s_{i}^{-} / D^{-}$for $i=1,2$ and the variables $\Gamma_{z}$ and $\Gamma_{v}$ represent $X_{u}(\xi)$ and $X_{v}(\xi)$ respectively. Similarly, on the interval $(\xi, 1]$,

$$
\begin{aligned}
& X_{\nu}(x)=\frac{1}{s_{2}^{+}-s_{1}^{+}}\left(\frac{\left(\Gamma_{u}+s_{1}^{+} \Gamma_{v}\right) s_{2}^{+}}{\cos (1-\xi) \alpha_{1}^{+}} \cos \alpha_{1}^{+}(1-x)-\frac{\left(\Gamma_{u}+s_{2}^{+} \Gamma_{v}\right) s_{1}^{+}}{\cos (1-\xi) \alpha_{2}^{+}} \cos \alpha_{2}^{+}(1-x)\right), \\
& X_{v}(x)=\frac{1}{s_{2}^{+}-s_{1}^{+}}\left(\frac{\Gamma_{u}+s_{2}^{+} \Gamma_{v}}{\cos (1-\xi) \alpha_{2}^{+}} \cos \alpha_{2}^{+}(1-x)-\frac{\Gamma_{u}+s_{1}^{+} \Gamma_{v}}{\cos (1-\xi) \alpha_{1}^{+}} \cos \alpha_{1}^{+}(1-x)\right) .
\end{aligned}
$$

By design the solutions (2.9) are continuous at $x=\xi$. In addition, however, they must also satisfy the conditions for continuity of flux at this point, namely

$$
\lim _{x \rightarrow \xi^{-}} X_{u}^{\prime}(x)=\lim _{x \rightarrow \xi^{+}} X_{u}^{\prime}(x) \text { and } \lim _{x \rightarrow \xi^{-}} D^{-} X_{0}^{\prime}(x)=\lim _{x \rightarrow \xi^{+}} D^{+} X_{0}^{\prime}(x) .
$$

Substituting the solutions (2.9) into these limits, we obtain the following simultaneous equations for $\Gamma_{u}$ and $\Gamma_{0}$ :

$$
P(\lambda) \Gamma_{u}+Q(\lambda) \Gamma_{v}=0, \quad R(\lambda) \Gamma_{u}+S(\lambda) \Gamma_{v}=0,
$$

where

$$
\begin{aligned}
& P(\lambda)=\left(s_{1}^{-} T_{2}^{-}-s_{2}^{-} T_{1}^{-}\right) /\left(s_{2}^{-}-s_{1}^{-}\right)+\left(s_{1}^{+} T_{2}^{+}-s_{2}^{+} T_{1}^{+}\right) /\left(s_{2}^{+}-s_{1}^{+}\right), \\
& Q(\lambda)=s_{1}^{-} s_{2}^{-}\left(T_{2}^{-}-T_{1}^{-}\right) /\left(s_{2}^{-}-s_{1}^{-}\right)+s_{1}^{+} s_{2}^{+}\left(T_{2}^{+}-T_{1}^{+}\right) /\left(s_{2}^{+}-s_{1}^{+}\right), \\
& R(\lambda)=D^{-}\left(T_{2}^{-}-T_{1}^{-}\right) /\left(s_{2}^{-}-s_{1}^{-}\right)+D^{+}\left(T_{2}^{+}-T_{1}^{+}\right) /\left(s_{2}^{+}-s_{1}^{+}\right), \\
& S(\lambda)=D^{-}\left(s_{1}^{-} T_{1}^{-}-s_{2}^{-} T_{2}^{-}\right) /\left(s_{2}^{-}-s_{1}^{-}\right)+D^{+}\left(s_{1}^{+} T_{1}^{+}-s_{2}^{+} T_{2}^{+}\right) /\left(s_{2}^{+}-s_{1}^{+}\right),
\end{aligned}
$$

and $T_{j}^{-}=\alpha_{j}^{-} \tan \xi \alpha_{j}^{-}$and $T_{j}^{+}=\alpha_{j}^{+} \tan (1-\xi) \alpha_{j}^{+}$for $j=1,2$. From the solutions (2.9), $\Gamma_{u}=\Gamma_{v}=0$ implies $X_{u} \equiv X_{v} \equiv 0$. Hence, for nontrivial solutions in $X_{u}$ and $X_{v}$, we require

$$
F(\lambda) \equiv P(\lambda) S(\lambda)-Q(\lambda) R(\lambda)=0 .
$$

Here $F(\lambda)$ represents the dispersion relation relating the rate of growth of linear instabilities to a given set of parameter values. The Turing space is therefore described by the inequalities (2.2a) and (2.2b) and the conditions that guarantee a solution of (2.11) with positive real part. Note that in deriving the dispersion relation (2.11) we have assumed that each of $s_{1}^{-}-s_{2}^{-}$, and $\cos \xi \alpha_{j}^{-}$and $\cos (1-\xi) \alpha_{j}^{-}$for $j=1$ or 2 , are nonzero. Under these conditions, for $D^{-} \neq D^{+}$, the solutions for $u$ and $v$ cannot, in general, satisfy continuity of flux at $x=\xi$. Hence, except in very special circumstances, our assumptions are valid. 


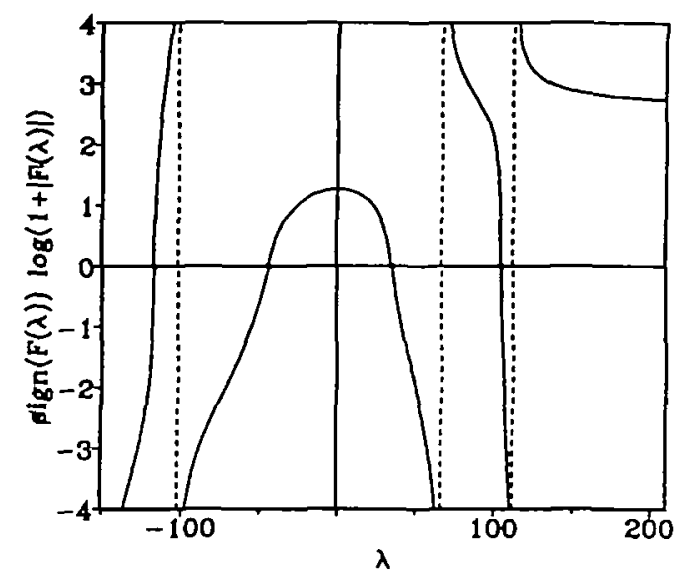

FIG. 2. A typical functional form of the dispersion relation $F(\lambda)$, defined in (2.11), for Schnackenberg kinetics (3.1), with parameter values $\lambda=1040, A=0.1, B=0.9, D^{-}=7, D^{+}=12 \xi=0.5$. To better illustrate the infinities of $F(\lambda)$, we plot sign $(F(\lambda)) \log (1+|F(\lambda)|)$ on the vertical axis. For this particular set of parameters there are two unstable modes, with approximate linear growth rates $\lambda=34.9$ and $\lambda=104.4$. For all parameter values, a straightforward calculation shows that $F(\lambda)=O(\lambda)$ as $\lambda \rightarrow \infty$.

In general, solutions of (2.11) will be complex valued. However, for homogeneous diffusion coefficients, all roots of the dispersion relation with positive real part can be shown to be real valued. In the inhomogeneous case, extensive numerical simulations of the full nonlinear system show that the spatially uniform solution always evolves to a steady state rather than a temporally oscillating solution, suggesting that the uniform solution bifurcates through real eigenvalues. Therefore we restrict our investigations to real values of $\lambda$. In contrast to the homogeneous case, the dispersion relation (2.11) cannot be solved analytically when $D^{-} \neq D^{+}$. However, straightforward algebra shows that whenever $\lambda$ is real, $F(\lambda)$ is real valued and therefore amenable to simple numerical calculation. Typical forms of the dispersion relation are illustrated in Fig. 2.

\section{Numerical solutions of the nonlinear model}

To apply the analysis of the previous section, we take, as a specific example, the simplest of the family of cross activator-inhibitor models proposed by Schnackenberg (1979). When appropriately nondimensionalized in one space dimension with a step function in the diffusion coefficient of the activator chemical $v$, the model equations take the form

$$
\begin{aligned}
& \frac{\partial u}{\partial t}=\frac{\partial^{2} u}{\partial x^{2}}+\gamma\left(A-u+u^{2} v\right), \\
& \frac{\partial v}{\partial t}=\frac{\partial}{\partial x}\left(D(x) \frac{\partial v}{\partial x}\right)+\gamma\left(B-u^{2} v\right),
\end{aligned}
$$

for $x \in(0,1)$. Here $D(x)$ is the step function defined by $(2.5), \gamma$ is the scale factor, 


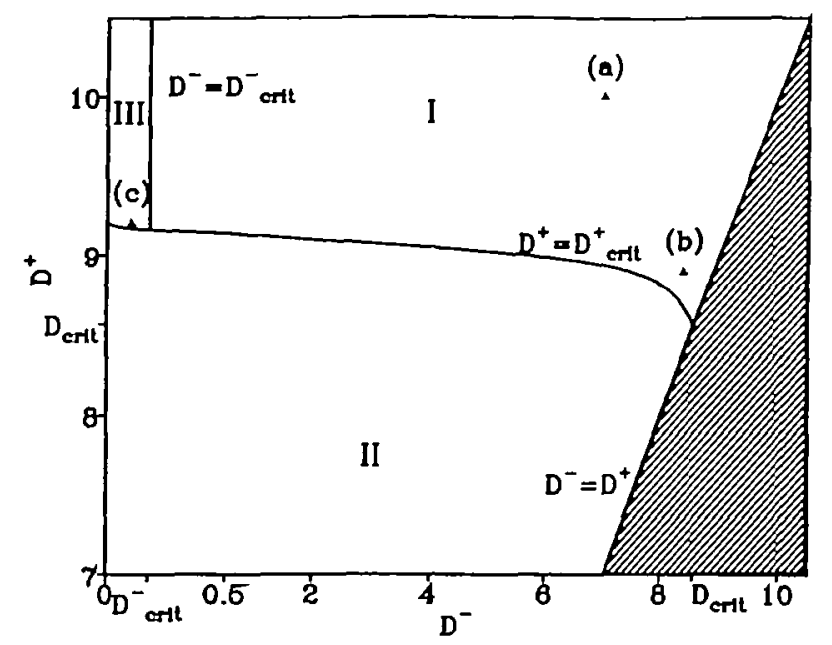

FlG. 3. The parameter space for the reaction-diffusion system (3.1). The diffusion coefficient of the species $u$ is constant throughout the domain, while that of $v$ has the constant value $D^{-}$on $[0, \xi)$ and $D^{+}$on $(\xi, 1]$. The region of interest is $D^{-}<D^{+}$; the shaded region is therefore ignored. In region $I$, patterns are either type $B$ isolated patterns or (for larger values of $D^{ \pm}$) nonisolated patterns; the division between these types is arbitrary. In region II, $D^{+}<D_{\text {crl }}^{+}$and thus the system does not exhibit diffusion-driven instability. For parameters in region III, diffusion-driven instability gives rise to type $A$ isolated patterns (see text for details). Parameter values are $\gamma=1040, \xi=0.5, A=0.1, B=0.9$. Solutions at the points indicated are shown in Fig. 4. To better illustrate the behaviour for $D^{-}$small, we use a magnified linear horizontal scale on $0 \leqslant D^{-} \leqslant 0.5$.

and $A$ and $B$ are positive constants. We impose zero-flux boundary conditions. This system has a unique homogeneous steady state, at $u=A+B, v=B /(A+B)^{2}$, which is stable to homogeneous perturbations provided $(A+B)^{3}>B-A$; all numerical simulations we present use values of $A$ and $B$ satisfying these inequalities.

Using the dispersion relation (2.11) we can determine numerically the parameter space in which this system exhibits diffusion-driven instability. As expected intuitively, diffusion-driven instability is observed whenever both $D^{-}$and $D^{+}$exceed $D_{\text {crit }}$, where $D_{\text {crit }}$ is the critical value which $D$ must exceed for diffusion-driven instability in the homogeneous case $D^{+}=D^{-}=D$. Moreover, our results imply that when $D^{-}$lies below $D_{\text {crit }}$ in $[0, \xi)$, the value of $D^{+}$must exceed some critical value $D_{\text {crit }}^{+}$which is larger than $D_{\text {crit }}$, in $(\xi, 1]$. The variation of $D_{\text {crit }}^{+}$with $D^{-}$is illustrated in Fig. 3. We thus define the Turing space by $(2.2 \mathrm{a}, \mathrm{b})$ and the following conditions:

(1) $D^{+}>D^{-}>D_{\text {crit }}$,

(2) $D^{-}<D_{\text {crit }}$ and $D^{+}>D_{\text {crit }}^{+}>D_{\text {crit }}$.

It is important to note that these conditions depend crucially on the fact that $a>0$. For $a<0$ the inequalities are essentially reversed so that the Turing space is defined by the conditions (2.2a, b), $D_{\text {crit }}>D^{+}>D^{-}, D^{+}>D_{\text {cril }}$, and $D_{\text {crit }}>D_{\text {crit }}^{-}>D^{-}$.

Figure 4 shows that the key qualitative features of the patterns generated by the full nonlinear model (3.1) are generally captured by the solutions of the linearized 

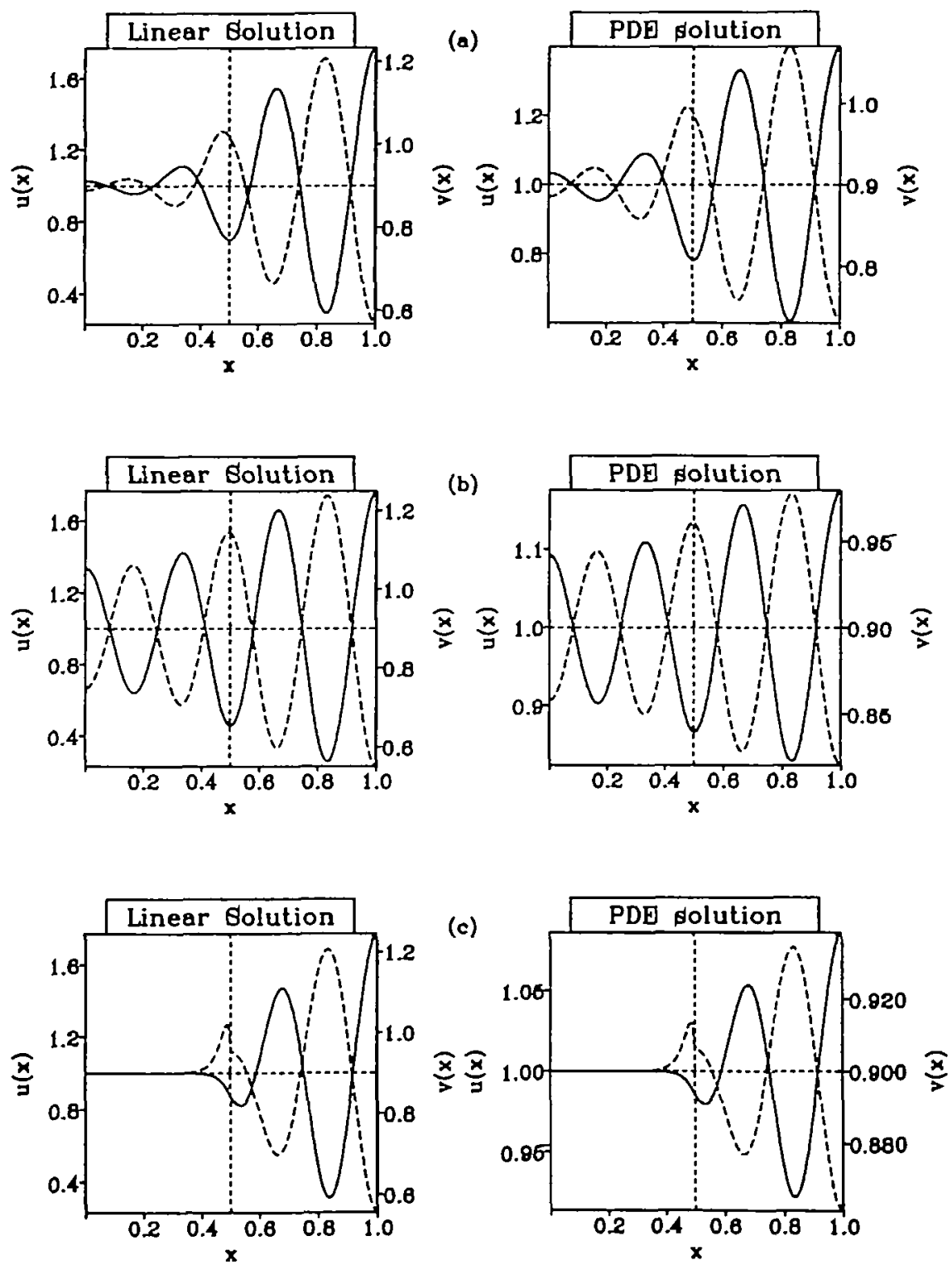

Fig. 4. Comparison of the steady-state solution profiles of the Schnackenberg system (3.1) with the linear solution (2.9) in different regions of parameter space for initial conditions of small random perturbations about the uniform steady state: (a) a type-B isolated pattern, with $D^{-}=7$ and $D^{+}=10$; (b) a nonisolated pattern, with $D^{-}=8.4$ and $D^{+}=8.9$; (c) a type-A isolated pattern, with $D^{-}=0.1$ and $D^{+}=9.2$. The other parameter values are $A=0.1, B=0.9, \gamma=1040, \xi=0.5$. The location of these solutions in the $D^{-}-D^{+}$parameter space is illustrated in Fig. 3. As the morphogen concentrations grow, linear analysis is no longer valid. However, in each case the qualitative behaviour of the full nonlinear pattern is captured by the linear solution. For sufficiently large $D^{+}$, the uniform steady state becomes unstable to several modes with similar growth rates, and different initial conditions result in qualitatively different patterns. Note that the patterns for $u$ and $v$ are out of phase $(u:-; v:---)$. 
system described by (2.9). As in the homogeneous case, when more than one solution of the linear system has a positive rate of growth, the nonlinear spatial pattern is, in many cases, well approximated by the solution corresponding to the largest real root of the dispersion relation (2.11). Moreover, this result is not specific to Schnackenberg kinetics, but is, in general, true for any cross or pure activator-inhibitor reaction diffusion model (Benson et al., 1992).

The crucial difference between the patterns illustrated in Fig. 4 and those obtained from reaction-diffusion models in a homogeneous environment is that, in the former case, pattern can be isolated in specific parts of the domain. By appropriately subdividing the domain any spatial pattern can be said to be restricted to a particular subdomain. However, in the inhomogeneous environment case, this restriction is independent of the scale parameter $\gamma$. This is in marked contrast to the homogeneous case, where varying the scale parameter alters the number and position of concentration peaks so that, for appropriate values of $\gamma$, pattern may occur in any subdomain.

Mathematically we can divide the isolated patterns of our model into two categories:

Type A: as the scale parameter $\gamma$ varies (but is large enough for diffusion-driven instability to occur), the steady-state solution profiles are always monotonic in one part of the domain, but in general oscillatory in space elsewhere.

Type B: as $\gamma$ varies, the steady-state solution profiles are generally oscillatory in space throughout the domain, but with much greater amplitude in one part of the domain than elsewhere.

Using the solutions (2.9) of the linearized model, one can derive conditions for the existence of type A isolated patterns in the Turing space (Benson et al., 1992). In particular, it can be shown that type A solutions, in which the pattern predominates in the interval $(\xi, 1]$, exist for sufficiently small values of the diffusion coefficient on $[0, \xi)$; specifically, for $D^{-}<D_{\text {crtt }}^{-}$, where $D_{\text {crit }}^{-}$is the smaller root of the equation $D^{2} a^{2}+2(2 b c-a d) D+d^{2}=0$.

\section{Smoothly varying diffusion coefficient}

In the above, we simply imposed a piecewise continuous spatial dependence on the diffusion coefficients in the reaction-diffusion model. This is, of course, a biologically unreasonable assumption. In this section, therefore, we consider how a realistic spatially varying coefficient could be set up. There are several ways in which this can be done, and here we focus on one possibility.

The composite model that we consider is one in which the spatial variation in diffusion coefficients is controlled by a regulatory chemical $c$. In our model we assume, as before, that the diffusion coefficient of $u$ is constant, but we now assume that the diffusion coefficient of $v$ is modulated by $c$. This could, for example, reflect an increase in gap junction permeability for $v$ due to the presence of $c$ (Othmer \& Pate, 1980).

We consider a mechanism for the production of $c$, in which $c$ is secreted at one end of the domain, diffuses and is degraded throughout the domain, but does not 
flow through the other boundary. The appropriate nondimensionalized equation for $c$ is

$$
c_{t}=v^{2} c_{x x}-\theta^{2} c
$$

subject to the boundary conditions

$$
c_{x}(0, t)=0, \quad c(1, t)=c_{0},
$$

where $v^{2}$ and $\theta^{2}$ are, respectively, the nondimensionalized diffusion coefficient and the rate of linear degradation of $c$.

If we assume that this reaction-diffusion equation reaches a stable equilibrium on a fast timescale during which insignificant changes in morphogen concentration take place, then the equilibrium distribution of $c$ is $c_{0} \cosh \delta x / \cosh \delta$, where $\delta=\theta / v$. A further assumption that the diffusivity of $u$ is independent of the concentration of $c$, while that of $v$ is directly proportional to it, implies that the ratio $D$ of diffusion coefficients now takes the form

$$
D(x)=\alpha c_{0} \cosh \delta x / \cosh \delta,
$$

where $\alpha$ is the constant of proportionality. Our model equations then become

$$
\begin{aligned}
& u_{t}=\gamma f(u, v)+u_{x x} \\
& v_{t}=\gamma g(u, v)+\left[D(x) v_{x}\right]_{x},
\end{aligned}
$$

where $D(x)$ is given by (4.3). Henceforth we shall refer to $D(x)$ as the diffusion coefficient for $v$, so that (4.3) represents a smoothly increasing diffusion coefficient for $v$. As before, we impose zero-flux boundary conditions on $u$ and $v$.

Although this system cannot be analysed using the method of linear analysis discussed in Section 2, it can be solved numerically. Figure 5 shows typical solutions for the Schnackenberg reaction kinetics. These simulations suggest that, as in the simple step function diffusion coefficient case, diffusion-driven instability occurs when either $D(x)$ exceeds $D_{\text {crit }}$ everywhere in the domain, or $D(0)=\alpha c_{0} / \cosh \delta$ is less than $D_{\text {crit }}$ and $D(1)=\alpha c_{0}$ is greater than some critical value $D(1)_{\text {crit }}$, where $D(1)_{\text {crit }}>D_{\text {crit }}$

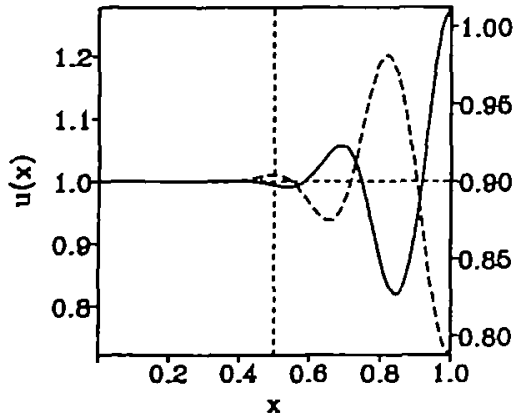

(a)

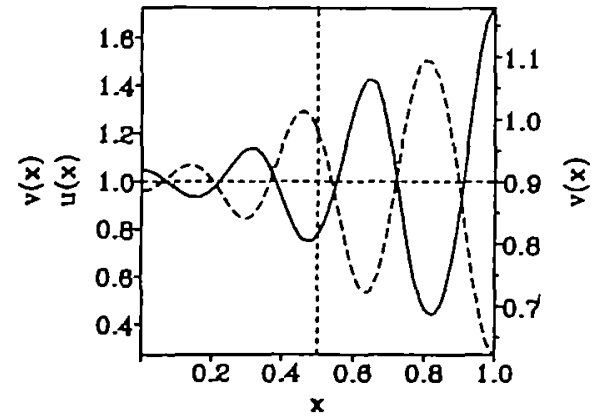

(b)

FIG. 5. (a) and (b): Solutions of (4.1)-(4.4) for Schnackenberg reaction kinetics with parameter values $A=0.1$ and $B=0.9$. With $\alpha=1, D(x)=c_{0} \cosh \delta x / \cosh \delta$, where $\delta$ and $c_{0}$ are chosen such that in (a) $D(0)=1$ and $D(1)=15$ and in (b) $D(0)=6$ and $D(1)=15$. The values of $\gamma$ are, respectively, 1000 and 2000 ( $u$ :- $; v:----$ ). 
and depends on $D(0), c_{0}, \alpha$, and $\delta$. (Here $D_{\text {crit }}$ is the critical diffusion coefficient that $D$ must exceed for pattern generation in the case of spatially homogeneous diffusion coefficients.) Moreover, for appropriate parameter values, the solutions of the model equations (4.4) with (4.3) reveal qualitatively similar patterns to those predicted by the simpler model analysed in Section 2 . In particular, patterns can be isolated in specific parts of the domain, and where the solution is oscillatory in space, the pattern does not have a constant amplitude of oscillation. This phenomenon does not depend critically on the steady-state distribution of $c$; other smoothly varying distributions produce similar results. Our analysis for the simple step function in diffusion coefficient therefore provides a valuable understanding of how spatial inhomogeneities in dispersal terms affect pattern generation for a range of more biologically realistic systems.

\section{Biological applications}

We illustrate the application of the composite model by considering two experimental observations which contradict the predictions of the standard Turing model, namely the AP asymmetry of skeletal elements in the limb and the results of the experiments on double anterior limbs (Wolpert \& Hornbruch, 1990), which show that one can produce more complex skeletal patterns while keeping the domain size constant.

The results of Sections 2-4 show that reaction-diffusion systems with spatial variation in diffusion coefficients may produce isolated patterns and asymmetric oscillatory patterns. Moreover, they show that, in the composite model, the position and amplitude of peaks in concentration are influenced by the underlying spatial pattern in control chemical. In limb morphogenesis, therefore, the positional information supplied by the solution profiles could lead, via cell differentiation, to the specification of asymmetrically patterned elements, whose position within the domain would be controlled by the spatial distribution of $c$. This is in contrast to the standard Turing system with spatially uniform parameters, which produces identical and equally spaced elements. The patterns produced by (4.1)-(4.4) are therefore more consistent with those observed in certain developmental processes, for example digit patterning in the vertebrate limb, than those exhibited by the standard model. This is illustrated in Fig. 6 where, for a suitably chosen parameter set, the composite model exhibits a steady-state solution with three peaks in concentration which, in contrast to the standard model, have different amplitudes of oscillation. For a suitable choice of threshold concentration, our model would therefore specify three skeletal elements which are intrinsically distinct because of the varying concentrations of morphogen to which they are each exposed.

The asymmetry in the solution profiles of the composite model is a consequence of the spatially inhomogeneous background environment. Clearly, the developing chick limb bud is such an environment. At the posterior margin of the developing limb bud, known as the zone of polarizing activity, or ZPA (Saunders \& Gasseling, 1968), there is a group of specialized cells that controls skeletal patterning along the AP axis. When a donor ZPA is grafted to different positions along the AP axis of a host chick limb, growth along this axis is stimulated and additional digits are formed (Smith \& Wolpert, 1981). If we apply our composite model to this duplication 


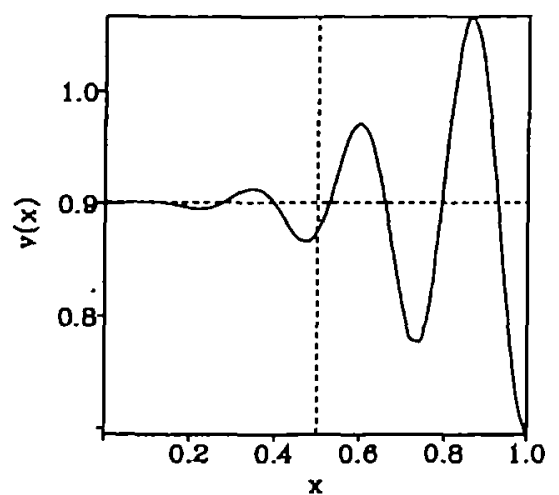

(a)

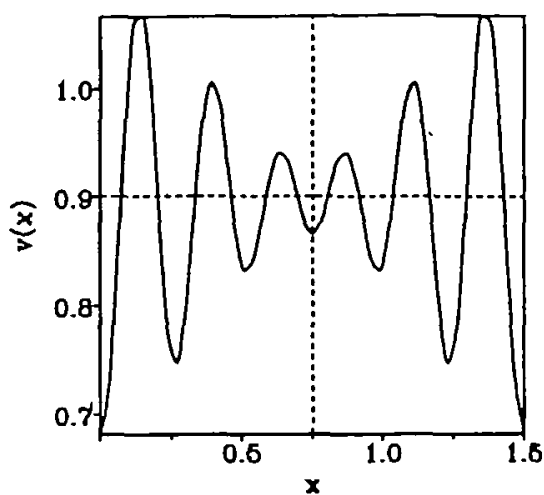

(b)

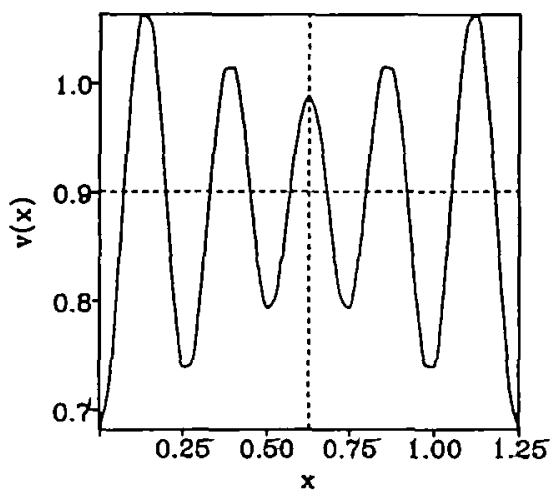

(c)

Fig. 6. (a) The solution of (4.1)-(4.4) on the domain $[0,1]$. (b, c) Solutions on the domain $[0, L]$ where $L=\frac{3}{3}$ and $\left\{\right.$, respectively, with boundary conditions $c(0, t)=c(L, t)=c_{0}$. In these cases the distribution of chemical concentration in $c$ is mirror symmetric. The corresponding diffusion coefficients for $v$ are, for $\alpha=1$, (a) $D(x)=c_{0} \cosh \delta x / \cosh \delta$, (b) $D(x)=c_{0} \cosh \delta\left(x-\frac{3}{4}\right) / \cosh \$ \delta$, and (c) $D(x)=$ $c_{0} \cosh \delta\left(x-\frac{\delta}{6}\right) / \cosh$ s. The values of $c_{0}$ and $\delta$ are chosen so that, in (a), $D(0)=6$ and $D(1)=12$; the other parameter values are $A=0.1, B=0.9$, and $\gamma=1700$. For a fixed threshold concentration (----), the prepattern in (a) specifies three elements which are intrinsically distinct. With this fixed single threshold concentration, (b) predicts a complete duplication of six elements, two of each kind specified in (a). The prepattern in (c), on a smaller domain, specifies only five elements. In both (b) and (c) the distribution of the elements is mirror symmetric about $x=\frac{1}{2}$.

experiment, and assume that the ZPA is the source of the regulatory chemical, $c$, then grafting a donor ZPA onto the anterior margin of the limb corresponds to creating a new source of $c$ at $x=0$. As this graft stimulates growth, the control chemical $c$ satisfies (4.1) with boundary conditions

$$
c(0, t)=c(0, L)=c_{0},
$$

where $L$ is the new domain length. The distribution of the control chemical, and hence the diffusion coefficient of $v$, is now given by

$$
D(x)=\alpha c_{0} \cosh \delta\left(x-\frac{1}{2} L\right) / \cosh \frac{1}{2} \delta L .
$$


For the case in which the domain increases by $50 \%$, Fig. 6 shows that the system can generate a mirror symmetric pattern which has six concentration peaks of varying amplitude. Together with the same threshold mechanism as before, the model predicts the formation of a duplicate set of additional digits, which is consistent with experimental results (see Maini \& Solursh, 1991, for a review of experimental results). On smaller domains (corresponding to transplanting donor ZPA to a more posterior site) the model predicts the formation of fewer digits (Fig. 6), as is also observed experimentally. Note that the standard Turing model also predicts that additional digits will form if the domain size is increased, but it cannot specify which digits are duplicated. In contrast, the composite model exhibits a prepattern in morphogen concentration which can distinguish between the different types of digit formed.

Another important property of the composite model is that spatial patterns may be restricted to specific parts of the domain. Figure 7 shows that, for certain parameter values, solution profiles have a single concentration peak isolated in one-half of the domain. In limb development, our model could therefore predict the formation of a single skeletal element which is specified asymmetrically along the AP axis. Moreover, as the position and number of concentration peaks is influenced by the distribution of the regulatory chemical $c$, spatial patterns specifying two skeletal structures can be produced by imposing symmetric boundary conditions (5.1) for $c$ and keeping the domain fixed in size. In this case, the composite model therefore predicts increases in pattern complexity without it being necessary to increase domain size. This is

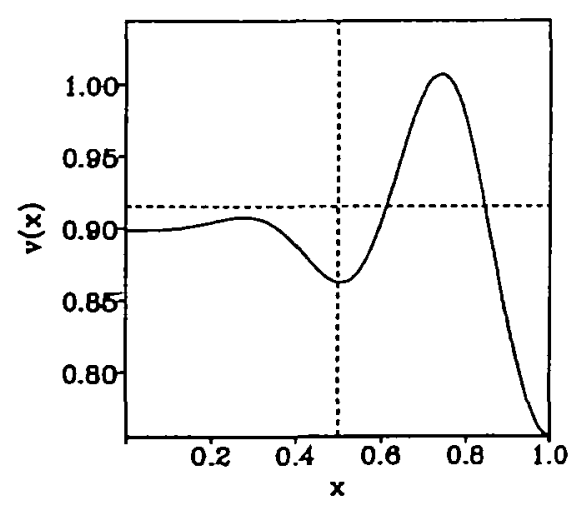

(a)

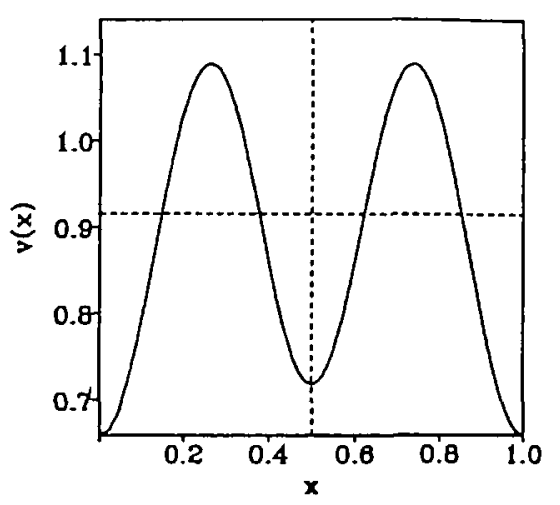

(b)

FIG. 7. (a) The solution of the coupled partial differential equation system (4.1)-(4.4) for Schnackenberg kinetics with parameter values $A=0.1, B=0.9$, and $\gamma=1040$. For simplicity, the constant of proportionality $\alpha$ is set equal to 1 . Thus, the diffusivity function $D(x)$ has the form $c_{0} \cosh \delta x / \cosh \delta$. Here $c_{0}$ and $\delta$ are chosen so that $D(0)=2$ and $D(1)=14$. (b) Solution of the model equations (4.4) for a prepattern in diffusivity which is symmetric about $x=1$ : In this case, the diffusion coefficient $D(x)$ is identical to that in (a) in the interval $\left(\frac{1}{2}, 1\right] ; D(x)$ is $c_{0} \cosh \delta(1-x) / \cosh \delta$ in $\left[0, \frac{1}{2}\right)$ and $c_{0} \cosh \delta(1-x) / \cosh \delta$ in $\left(\frac{1}{2}, 1\right]$. All other parameter values are unaltered. For a suitable choice of the threshold concentration (-- - ) the prepattern in (a) may specify a single structure in the interval $\left[\frac{1}{2}, 1\right)$, and the prepattern in (b) may specify two elements symmetrically placed about $x=\frac{1}{2}$. In the two-step model, therefore, one can generate more complex patterns without changing domain size or parameter values. This is in contrast to the standard Turing model wherein patterns are entirely scale dependent. 
consistent with the results of recent experiments (Wolpert \& Hornbruch, 1990) which show that double anterior composite limbs give rise to two humeral elements without there being a significant increase in the normal length of the AP axis. Note that in the standard Turing model one would predict, at least for low mode numbers, that the pattern complexity would remain unchanged for a fixed domain size (Dillon et al., 1992).

\section{Discussion}

Development of spatial pattern in the early embryo results from the interaction of several processes in a complex hierarchy of mechanisms. Most models for morphogenesis to date have, however, focused on a particular mechanism. Although such models are capable of capturing some aspects of development, they are inconsistent with key experimental observations. In this paper we have analysed a two-step hierarchy of patterning mechanisms in which the spatial pattern of a control chemical regulates morphogen diffusivity in the overlying reaction-diffusion mechanism. We have shown that such a system can produce isolated asymmetric spatial patterns, and that it is capable of producing patterns of increased complexity without a change in domain size. These properties are consistent with experimental observations that contradict the standard Turing model.

In both our model and the standard Turing model, the polarity of certain solutions is determined by the initial conditions. This is, of course, a drawback as most developmental processes are robust. Recently, Dillon et al. (1992) have shown that imposing the appropriate boundary conditions on a reaction-diffusion system can lead to more robust pattern generation. In our framework, this specialized differentiation of the boundary cells constitutes another level in the developmental hierarchy.

\section{Acknowledgements}

DLB acknowledges the Wellcome Trust for a Prize Studentship in Mathematical Biology. JAS was supported by a Junior Research Fellowship at Merton College, Oxford.

This paper was read at the Sixth IMA Conference on the Mathematical Theory of the Dynamics of Biological Systems, held in Oxford, 1-3 July 1992.

\section{REFERENCES}

AuchmuTy, J. F. G., \& Nicholis, G. 1975 Bifurcation analysis of nonlinear reaction-diffusion equations-I. Evolution equations and the steady state solutions. Bull. Math. Biol. 37, 323-65.

BARD, J. B. L:, \& LAUDER, I. 1974 How well does Turing's theory of morphogenesis work? J. Math. Biol. 45, 503-31. 
Benson, D. L., Sherratt, J. A., \& Main,, P. K. 1992 Diffusion driven instability in an inhomogeneous domain. Bull. Math. Biol. In press.

Brümmer, F., Zempel, G., Buhle, P., Stein, J.-C., \& Hulser, D. F. 1991 Retinoic acid modulates gap junctional permeability: A comparative study of dye spreading and ionic coupling in cultured cells. Exp. Cell Res. 196, 158-63.

CASTETS, V., Dulos, E., \& DE KEPPER, P. 1990 Experimental evidence of a sustained standing Turing-type non-equilibrium chemical pattern. Phys. Rev. Lett. 64, 2953-6.

Dillon, R., Maini, P. K., \& Othmer, H. G. 1992 Pattern formation in generalized Turing systems. I. Steady-state patterns in systems with mixed boundary conditions. Submitted.

GIERER, A., \& MEINHARDT, H. 1972 A theory of biological pattern formation. Kybernetika 12, 30-9.

HerschKowITZ-Kaufman, M. 1975 Bifurcation analysis of nonlinear reaction diffusion equations-II. Steady state solutions and comparison with numerical solutions. Bull. Math. Biol. 37, 589-636.

Hunding, A. 1987 Bifurcations in Turing systems of the second kind may explain blastula cleavage plane orientation. J. Math. Biol. 25, 109-21.

HundING, A. 1989 Turing prepatterns of the second kind simulated on supercomputer in three curvilinear coordinates and time. In: Cell to Cell Signalling: From Experiments to Theoretical Models (A. Goldbeter, ed.). New York: Academic Press, pp. 22935.

Hunding, A., Kauffman, S. A., \& GoOdwin, B. C. 1990 Drosophila segmentation: Supercomputer simulation of prepattern hierarchy. J. Theor. Biol. 145, 369-84.

Hunding, A., \& Sørenson, P. G. 1988 Size adaptation of Turing prepatterns. J. Math. Biol. 26, 27-39.

LACALLI, T. C. 1990 Modeling the Drosophila pair-rule pattern by reaction-diffusion: Gap input and pattern control in a 4-morphogen system. J. Theor. Biol. 144, 171-94.

MaINI, P. K., \& SoluRsh, M. 1991 Cellular mechanisms of pattern formation in the developing limb. Int. Rev. Cytol. 129, 91-133.

Metnhardt, H. 1982 Models of Biological Pattern Formation. London: Academic Press.

MuRray, J. D. 1989 Mathematical Biology. Heidelberg. Springer.

Noj, S., Nonho, T., Koyama, E., Kohki, M., Ohyama, K., Aoki, Y., Tamura, K., Ohsugi, K., IDE, H., TANIGUCHI, S., \& SAITo, T. 1991 Retinoic acid induces polarizing activity but is unlikely to be a morphogen in the chick limb bud. Nature 350, 83-6.

OTHMER, H. G., \& PATE, E. 1980 Scale-invariance in reaction-diffusion models of spatial pattern formation. Proc. Natl. Acad. Sci. USA 77, 4180-4.

OuYANG, Q., \& SWINNEY, H. L. 1991 Transition from a uniform state to hexagonal striped Turing patterns. Nature 352, 610-12.

SAUNDERS, J. W., \& GASSELING, M. T. 1968 Ectodermal-mesenchymal interactions in the origin of limb symmetry. In: Epithelial-Mesenchymal Interactions (P. Fleischmajer \& R. E. Billingham, eds.). Baltimore, MD: Williams and Wilkins, pp. 78-97.

SCHNACKENBERG, J. 1979 Simple chemical reaction systems with limit cycle behaviour. J. Theor. Biol. 81, 389-400.

SMITH, J. C., \& WOLPERT, L. 1981 Pattern formation along the anteroposterior axis of the chick limb: The increase in width following a polarizing graft and the effect of $\mathrm{x}$-irradiation. J. Embryol. Exp. Morph. 63, 127-44.

Thaller, C., \& Eichele, G. 1987 Identification and spatial distribution of retinoids in the developing chick limb. Nature 327, 625-8.

Turrng, A. M. 1952 The chemical basis of morphogenesis. Phil. Trans. R. Soc. Lond. B 237, $37-72$.

Walbot, V., \& Holder, N. 1987 Developmental Biology. New York: Random House.

Wanex, N., Gardiner, D. M., Muneoka, K., \& Bryant, S. V. 1991 Conversion by retinoic acid of anterior cells into ZPA cells in the chick wing bud. Nature 350, 81-3.

WOLPERT, L. 1969 Positional information and the spatial pattern of cellular differentiation. J. Theor. Biol. 25, 1-47. 
Wolpert, L. 1981 Positional information and spatial patterning. Phil. Trans. R. Soc. Lond. B 259, 441-50.

WOlPERT, L., \& Horniruch, A. 1990 Double anterior chick limb buds and models for cartilage rudiment specification. Development 109, 961-6. 
